\title{
Automatic brain MR image denoising based on texture feature-based artificial neural networks
}

\author{
Yu-Ning Chang and Herng-Hua Chang* \\ Computational Biomedical Engineering Laboratory (CBEL), Department of Engineering Science and \\ Ocean Engineering, National Taiwan University, Daan 10617 Taipei, Taiwan
}

\begin{abstract}
Noise is one of the main sources of quality deterioration not only for visual inspection but also in computerized processing in brain magnetic resonance (MR) image analysis such as tissue classification, segmentation and registration. Accordingly, noise removal in brain MR images is important for a wide variety of subsequent processing applications. However, most existing denoising algorithms require laborious tuning of parameters that are often sensitive to specific image features and textures. Automation of these parameters through artificial intelligence techniques will be highly beneficial. In the present study, an artificial neural network associated with image texture feature analysis is proposed to establish a predictable parameter model and automate the denoising procedure. In the proposed approach, a total of 83 image attributes were extracted based on four categories: 1) Basic image statistics. 2) Gray-level co-occurrence matrix (GLCM). 3) Gray-level run-length matrix (GLRLM) and 4) Tamura texture features. To obtain the ranking of discrimination in these texture features, a paired-samples t-test was applied to each individual image feature computed in every image. Subsequently, the sequential forward selection (SFS) method was used to select the best texture features according to the ranking of discrimination. The selected optimal features were further incorporated into a back propagation neural network to establish a predictable parameter model. A wide variety of MR images with various scenarios were adopted to evaluate the performance of the proposed framework. Experimental results indicated that this new automation system accurately predicted the bilateral filtering parameters and effectively removed the noise in a number of MR images. Comparing to the manually tuned filtering process, our approach not only produced better denoised results but also saved significant processing time.
\end{abstract}

Keywords: Image feature, image texture, image denoising, neural network, MRI

\section{Introduction}

Magnetic resonance imaging (MRI) is one powerful apparatus used to investigate the structure and function of the brain in both health and disease. It has been one of the most frequently used neuroimaging modalities due to its high contrast among different soft tissues, high spatial resolution across the entire field of view, and multi-spectral characteristics. However, random noise inevitably appears during the acquisition process that includes eddy-current distortions, physiological motion and

\footnotetext{
* Address for correspondence: Herng-Hua Chang, Computational Biomedical Engineering Laboratory (CBEL), Department of Engineering Science and Ocean Engineering, National Taiwan University, Daan 10617 Taipei, Taiwan. Tel.: +886-2-3366-5745; Fax: +886-2-2392-9885; E-mail: herbertchang@ntu.edu.tw.
} 
instabilities of the MRI scanning hardware. The noise not only affects the medical diagnostic tasks but also degrades many computerized image processing and analysis procedures such as tissue classification, visualization, super-resolution, segmentation, and registration. Consequently, noise removal or reduction is important and essential to maintain the quality of brain MR images for a wide variety of subsequent applications [1].

For the past decades, Gaussian filters have been widely used in many MR image processing applications for its simplicity [2]. This filter computes a weighted average of pixel values in the neighborhood in such a way that the weight decreases with distance from the kernel center. Although the Gaussian filter smoothes noise quite efficiently, edges are blurred significantly. To preserve the sharpness, a nonlinear method called the anisotropic diffusion filter [3] has been proposed. In their approach, pixel values are averaged from neighborhoods, whose size and shape depend on local image variation that is measured at every point [4]. Alternatively, one promising technique that attempted to improve the Gaussian filter is the bilateral filter [5]. It has been shown that the bilateral filter performed effectively in MR image noise suppression and it has been the subject of many further studies [6-8].

Nevertheless, most denoising algorithms require laborious tuning of parameters that are often sensitive to specific image features and textures. Automation of these parameters through artificial intelligence techniques will be highly beneficial $[8,9]$. This paper proposes to automate the denoising process using an artificial neural network based on image texture feature analysis. For its effectiveness and simplicity, the bilateral filter will be adopted as the key denoising algorithm in the automation framework.

\section{Methods}

The proposed system consists of two major phases: training and testing, as illustrated in Figure 1. The intention is to establish a predictable parameter model for the values of the parameters: $N, \sigma_{S}$, and $\sigma_{R}$ in the bilateral filter, which is explained in Section 2.3. In both phases, the image texture feature computation plays an important role in the automation process as described subsequently.

\subsection{Texture feature extraction}

Texture in an image refers to the appearance, structure and arrangement of an object within the image that describes the spatial variations of different pixels. It has long been recognized that texture

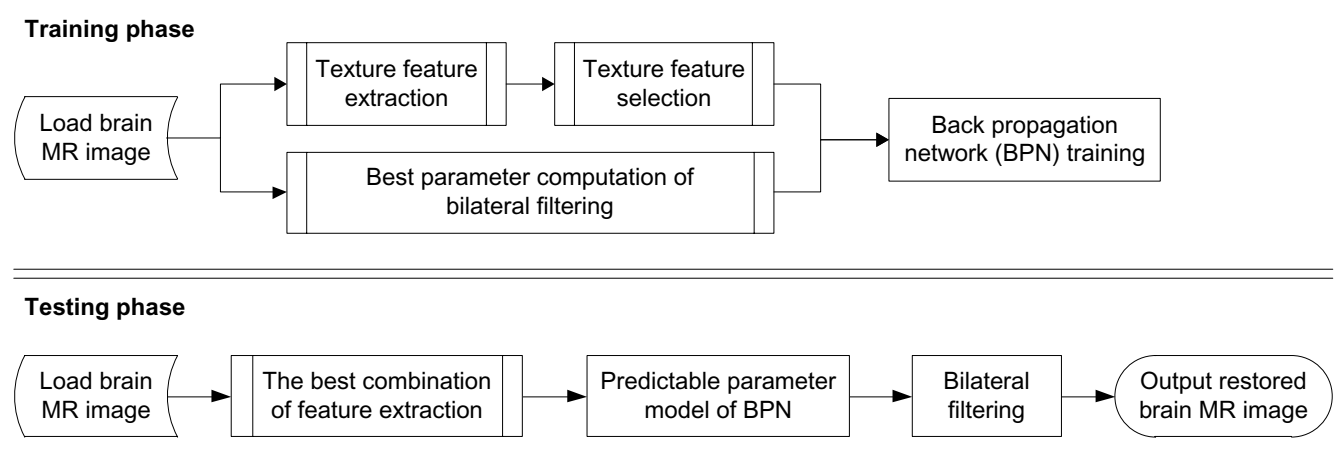

Fig. 1. Flowchart of the proposed automatically denoising scheme. 


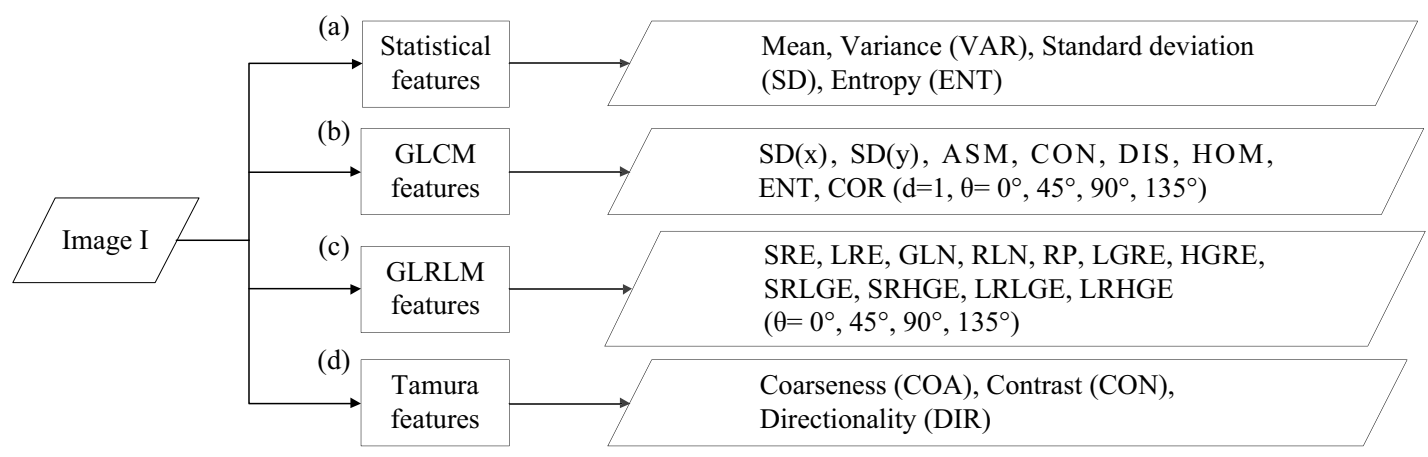

Fig. 2. Image texture feature categories: (a) basic statistics, (b) GLCM, (c) GLRLM and (d) Tamura features.

features play an important role in a wide variety of computer vision, image analysis and pattern recognition applications. In particular, texture features have been extensively used in medical images to quantify image properties such as homogeneity, contrast, and regularity [10-12].

In our approach, a total of 83 image features are obtained based on four different feature extraction categories as shown in Figure 2. In each individual image, there are four basic statistical features computed directly from the image intensity, 32 features in the gray-level co-occurrence matrix (GLCM) [13-15] class including eight basic features multiplied by four directions, 44 features in the gray-level run-length matrix (GLRLM) [14, 16, 17] class including 11 basic features multiplied by four directions, and three complex features in the Tamura texture feature class[18-20].

\subsection{Feature selection}

\subsubsection{Paired-samples t-test}

To facilitate the feature selection process and to obtain the ranking of discrimination among the texture features, a paired-samples t-test [21,22] is then applied to each individual image feature to evaluate the discrimination ability in two categories: noise level and slice position. The evaluation is based on the distinguishing ability between noise levels, intensity distributions, and anatomical geometries in two images according to the average $p$-value. Those features with an average $p<0.05$ are selected as candidates for the subsequent process [23].

\subsubsection{Sequential forward selection (SFS)}

If a large amount of features are selected, the computation of the neural network training will be very time-consuming. Moreover, redundant inputs may lead to degraded performance. Consequently, it is essential to select the most relevant attributes that are beneficial to the proposed automatically denoising scheme while removing the redundant attributes. To speed up the computation of the neural network, a sequential forward selection (SFS) method [24] is used to obtain the minimal subset of the image texture features based on the ranking of discrimination from the paired-samples t-test results.

The selection procedure starts by initializing an empty set $Y_{0}$ that is considered as the best subset of the features. The feature $f^{+}$that gives the lowest average p-value has the highest priority to be added into $Y_{k}$ at each step $k$. The selected features are then used in the neural network to obtain the prediction accuracy $A$ based on a ten-fold cross validation method [25]. The overall procedure of the sequential selection algorithm is described as follows:

- Start with the empty set $Y_{0}=\{\varnothing\}$ 
- Add in the next best feature $f^{+}=\arg \min _{f^{+} \notin Y_{k}}[F(p)]$

- If $A\left(Y_{k}+f^{+}\right)>A\left(Y_{k}\right)$

3.1 Update $Y_{k+1}=Y_{k}+f^{+} ; k=k+1$

3.2 Go to step 2

- End

\subsection{Best parameter computation of bilateral filtering}

The essence of the bilateral filter [5] is to combine both geometric closeness in the spatial domain and gray value similarity in the range domain as a nonlinear filter for image denoising. More specifically, let $\left(\theta_{x}, \theta_{y}\right)$ be the location of the pixel centered at a $(2 N+1) \times(2 N+1)$ neighborhood and $\Psi_{\theta_{x}, \theta_{y}}$ is the pixels in the neighborhood of $\left(\theta_{x}, \theta_{y}\right)$ defined as

$$
\Psi_{\theta_{x}, \theta_{y}}=\left\{\left(\mu_{x}, \mu_{y}\right):\left(\mu_{x}, \mu_{y}\right) \in\left[\theta_{x}-N, \theta_{y}+N\right] \times\left[\theta_{x}-N, \theta_{y}+N\right]\right\}
$$

The weighting functions for the spatial component and the radiometric component are defined respectively in Eqs. (2) and (3) as

$$
\begin{aligned}
& W_{\theta_{x}, \theta_{y}}^{S}\left(\mu_{x}, \mu_{y}\right)=\exp \left[-\frac{\left|\left(\mu_{x}, \mu_{y}\right)-\left(\theta_{x}, \theta_{y}\right)\right|^{2}}{2 \sigma_{S}^{2}}\right] \\
& W_{\theta_{x}, \theta_{y}}^{R}\left(\mu_{x}, \mu_{y}\right)=\exp \left[-\frac{\left|I\left(\mu_{x}, \mu_{y}\right)-I\left(\theta_{x}, \theta_{y}\right)\right|^{2}}{2 \sigma_{R}^{2}}\right]
\end{aligned}
$$

where $I(\because$,$) is the intensity value at the given position (\because)), \sigma_{S}$ and $\sigma_{R}$ are the controlling parameters used to adjust $W^{S}$ and $W^{R}$, respectively. The ensemble weight in the bilateral filter is then the product of $W^{S}$ and $W^{R}$ as given in Eq. (4).

$$
W_{\theta_{x}, \theta_{y}}\left(\mu_{x}, \mu_{y}\right)=W_{\theta_{x}, \theta_{y}}^{S}\left(\mu_{x}, \mu_{y}\right) W_{\theta_{x}, \theta_{y}}^{R}\left(\mu_{x}, \mu_{y}\right)
$$

In practice, each pixel is filtered using normalized weights to obtain the final filtered image using

$$
\tilde{I}\left(\theta_{x}, \theta_{y}\right)=\frac{\sum_{\left(\mu_{x}, \mu_{y}\right) \in \Psi} W_{\theta_{x}, \theta_{y}}\left(\mu_{x}, \mu_{y}\right) I\left(\mu_{x}, \mu_{y}\right)}{\sum_{\left(\mu_{x}, \mu_{y}\right) \in \Psi} W_{\theta_{x}, \theta_{y}}\left(\mu_{x}, \mu_{y}\right)}
$$

In the best parameter computation procedure as shown in Figure 1, the intention is to obtain the optimal values of $N, \sigma_{S}$, and $\sigma_{R}$ in the bilateral filter for the subsequent training process through a brute-force method based on the peak signal-to-noise ratio (PSNR), which is defined as

$$
\text { PSNR }=10 \cdot \log _{10}\left[\frac{L_{i} L_{j} I_{\text {max }}{ }^{2}}{\sum_{i=1}^{L_{i}} \sum_{j=1}^{L_{j}}\left|I^{\prime}(i, j)-I(i, j)\right|^{2}}\right]
$$

where $I$ represents the original $L_{i} \times L_{j}$ image, $I^{\prime}$ represents the restored image, and $I_{\max }$ equals to 255 for 8-bit grayscale cases. The higher the PSNR values the better the restoration results. These optimal 
values of the three parameters are then used in the learning stage to train the neural network system as well as for the evaluation of the automatic noise removal scheme.

\subsection{Back propagation network (BPN)}

The back propagation network (BPN) [26-28] with multilayer feedforward and error back propagation is adopted in both phases of the proposed noise removal scheme associated with the selected texture features. The famous Levenberg-Marquardt (LM) learning algorithm $[29,30]$ is used in the automation system for the training purpose.

\section{Experimental results}

T1-weighted MR image volumes obtained from the BrainWeb [31] dataset with normal and multiple sclerosis (MS) were used for the experiments. For each type, there are five different slice thicknesses, six noise levels and three intensity non-uniformities. The prediction accuracy is measured using the mean absolute percentage error (MAPE) as

$$
\text { MAPE }=\frac{1}{M} \sum_{k=1}^{M}\left|\frac{x(k)-x^{\prime}(k)}{x(k)}\right| \times 100 \%
$$

where $x(k)$ represents the expected value, $x^{\prime}(k)$ represents the predicted (output) value, and $M$ is the number of data being tested. The smaller the value of MAPE, the better the prediction accuracy is. A total of $22501 \mathrm{~mm}$ normal MR slices were used for the training procedure.

Tables 1 and 2 present the order of significance based on the average p-value of the t-test results among all image texture features in noise level and slice position, respectively. After the SFS procedure, nine optimal features: SRLGE $\left(90^{\circ}\right)$, SRLGE $\left(45^{\circ}\right)$, SRLGE $\left(0^{\circ}\right)$, SRLGE $\left(135^{\circ}\right)$, COR $\left(45^{\circ}\right), \operatorname{COR}\left(135^{\circ}\right), \operatorname{COR}\left(0^{\circ}\right), \operatorname{RLN}\left(90^{\circ}\right)$, and COR $\left(90^{\circ}\right)$ were obtained, which were further used in the BPN training procedure. The MAPE score of the proposed parameter prediction system based on the ten-fold cross validation was $22.62 \%$. Figure 3 presents the restored image using the proposed automati cally denoising method on the $1 \mathrm{~mm}$ MS case. A high and consistent PSNR score of 30.56

Table 1

Paired-samples t-test results based on the p-value: noise level

\begin{tabular}{ll}
\hline$p$-value & Feature \\
\hline$[0.01,0.02)$ & $\operatorname{SRLGE}\left(45^{\circ}, 90^{\circ}\right)$ \\
{$[0.02,0.03)$} & $\operatorname{SRLGE}\left(0^{\circ}, 135^{\circ}\right), \operatorname{RLN}\left(90^{\circ}\right)$ \\
{$[0.03,0.04)$} & $\operatorname{RLN}\left(0^{\circ}\right), \operatorname{GLN}\left(0^{\circ}\right), \operatorname{CON}, \operatorname{RP}\left(0^{\circ}, 90^{\circ}\right), \operatorname{GLN}\left(45^{\circ}, 90^{\circ}\right), \operatorname{LGRE}\left(0^{\circ}\right), \operatorname{RLN}\left(135^{\circ}\right)$ \\
{$[0.04,0.05)$} & $\operatorname{GLN}\left(135^{\circ}\right), \operatorname{RLN}\left(45^{\circ}\right), \operatorname{RP}\left(45^{\circ}, 135^{\circ}\right), \operatorname{LGRE}\left(45^{\circ}, 90^{\circ}\right)$ \\
{$[0.05,0.07)$} & $\operatorname{LRHGE}\left(0^{\circ}, 45^{\circ}, 90^{\circ}, 135^{\circ}\right), \operatorname{LGRE}\left(135^{\circ}\right), \operatorname{HOM}\left(0^{\circ}, 45^{\circ}, 90^{\circ}, 135^{\circ}\right), \operatorname{CON}\left(90^{\circ}\right), \operatorname{DIR}, \operatorname{DIS}\left(0^{\circ}\right.$, \\
& $\left.90^{\circ}\right)$ \\
{$[0.07,0.09)$} & $\operatorname{DIS}\left(45^{\circ}, 135^{\circ}\right), \operatorname{LRE}\left(0^{\circ}, 45^{\circ}, 90^{\circ}\right), \operatorname{CON}\left(0^{\circ}, 45^{\circ}, 135^{\circ}\right), \operatorname{SRE}\left(0^{\circ}, 45^{\circ}\right), \operatorname{HGRE}\left(0^{\circ}, 45^{\circ}, 90^{\circ}\right)$, \\
& $\operatorname{SD}, \operatorname{VAR}\left(135^{\circ}\right), \operatorname{SRE}\left(90^{\circ}, 135^{\circ}\right), \operatorname{ENT}, \operatorname{LRE}\left(135^{\circ}\right), \operatorname{LRLGE}\left(0^{\circ}, 45^{\circ}, 90^{\circ}, 135^{\circ}\right), \operatorname{SRHGE}\left(0^{\circ}, 45^{\circ}\right.$, \\
{$[0.09,0.2)$} & $\operatorname{HGRE}\left(135^{\circ}, 135^{\circ}\right)$ \\
{$[0.2,0.4)$} & $\left.90^{\circ}, 135^{\circ}\right), \operatorname{ENT}\left(0^{\circ}, 45^{\circ}, 90^{\circ}, 135^{\circ}\right), \operatorname{SD}\left(\mathrm{x}, 0^{\circ}, 45^{\circ}, 90^{\circ}, 135^{\circ}\right), \operatorname{SD}\left(\mathrm{y}, 0^{\circ}, 45^{\circ}, 90^{\circ}, 135^{\circ}\right)$ \\
$\geq 0.4$ & $\operatorname{ASM}\left(0^{\circ}, 45^{\circ}, 90^{\circ}, 135^{\circ}\right)$ \\
\hline
\end{tabular}


Table 2

Paired-samples t-test results based on the p-value: slice position

\begin{tabular}{ll}
\hline$p$-value & Feature \\
\hline$[0.02,0.03)$ & $\operatorname{COR}\left(0^{\circ}, 45^{\circ}, 90^{\circ}, 135^{\circ}\right)$ \\
{$[0.03,0.04)$} & Mean, COA \\
{$[0.05,0.1)$} & $\operatorname{ASM}\left(0^{\circ}, 45^{\circ}, 90^{\circ}, 135^{\circ}\right)$ \\
{$[0.1,0.2)$} & $\operatorname{SD}\left(x, 0^{\circ}, 45^{\circ}, 90^{\circ}, 135^{\circ}\right), \operatorname{SD}\left(\mathrm{y}, 0^{\circ}, 45^{\circ}, 90^{\circ}, 135^{\circ}\right), \operatorname{ENT}\left(0^{\circ}, 45^{\circ}, 90^{\circ}, 135^{\circ}\right), \operatorname{LRLGE}\left(90^{\circ}\right)$, \\
& $\operatorname{VAR}, \operatorname{SD}, \operatorname{CON}\left(45^{\circ}, 135^{\circ}\right)$ \\
{$[0.2,0.3)$} & $\operatorname{LRLGE}\left(0^{\circ}, 45^{\circ}, 135^{\circ}\right), \operatorname{DIS}\left(0^{\circ}, 45^{\circ}, 90^{\circ}, 135^{\circ}\right), \operatorname{HOM}\left(0^{\circ}, 45^{\circ}, 135^{\circ}\right), \operatorname{SRE}\left(0^{\circ}, 45^{\circ}, 90^{\circ}, 135\right.$ \\
& $\left.{ }^{\circ}\right), \operatorname{LRE}\left(0^{\circ}, 45^{\circ}, 90^{\circ}, 135^{\circ}\right), \operatorname{CON}\left(0^{\circ}, 90^{\circ}\right), \operatorname{SRHGE}\left(45^{\circ}, 135^{\circ}\right), \operatorname{LRHGE}\left(90^{\circ}\right)$ \\
{$[0.3,0.4)$} & $\operatorname{HOM}\left(90^{\circ}\right), \operatorname{LRHGE}\left(0^{\circ}, 45^{\circ}, 135^{\circ}\right), \operatorname{ENT}, \operatorname{HGRE}\left(0^{\circ}, 45^{\circ}, 90^{\circ}, 135^{\circ}\right), \operatorname{SRHGE}\left(0^{\circ}, 90^{\circ}\right)$ \\
$\geq 0.4$ & $\operatorname{RP}\left(0^{\circ}, 45^{\circ}, 90^{\circ}, 135^{\circ}\right), \operatorname{LGRE}\left(0^{\circ}, 45^{\circ}, 90^{\circ}, 135^{\circ}\right), \operatorname{GLN}\left(0^{\circ}, 45^{\circ}, 90^{\circ}, 135^{\circ}\right), \operatorname{RLN}\left(0^{\circ}, 45^{\circ}\right.$, \\
& $\left.90^{\circ}, 135^{\circ}\right), \operatorname{DIR}, \operatorname{LGRE}\left(90^{\circ}\right), \operatorname{SRLGE}\left(0^{\circ}, 45^{\circ}, 90^{\circ}, 135^{\circ}\right), \operatorname{CON}$ \\
\hline
\end{tabular}

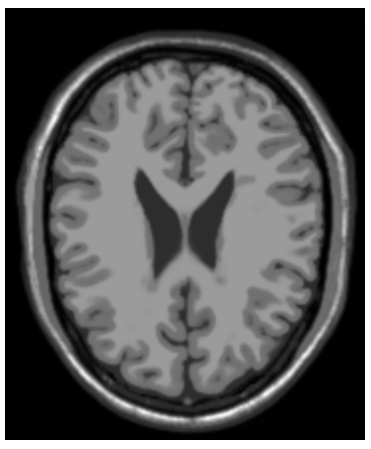

(a)

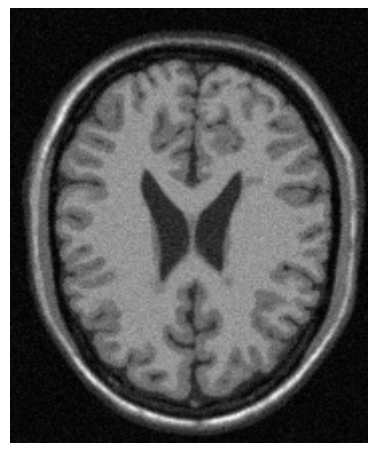

(b)

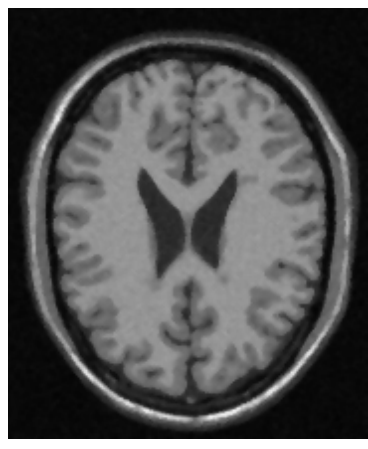

(c)

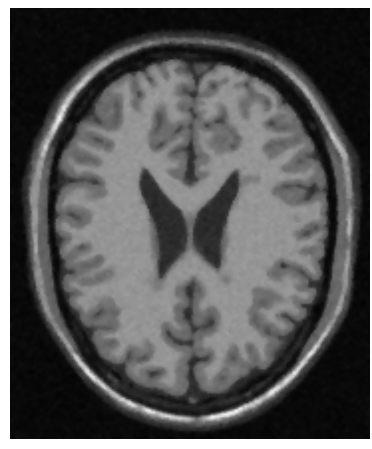

(d)

Fig. 3. Restoration results of the 96 slice in the $1 \mathrm{~mm}$ MS image volume: (a) intact image, (b) 5\% noisy image, (c) restored image of (b) with PSNR $=30.47$ by manually tuned bilateral filtering, (d) restored image of (b) with PSNR $=$ 30.56 using the proposed automatically denoising system.

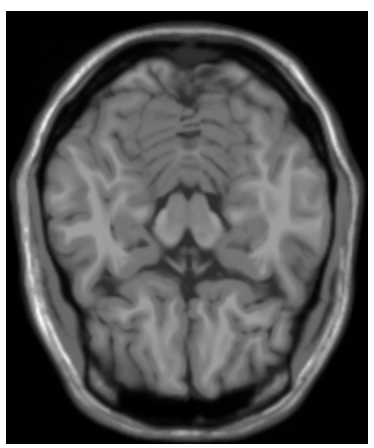

(a)

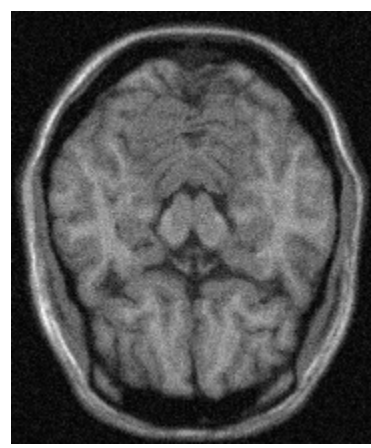

(b)

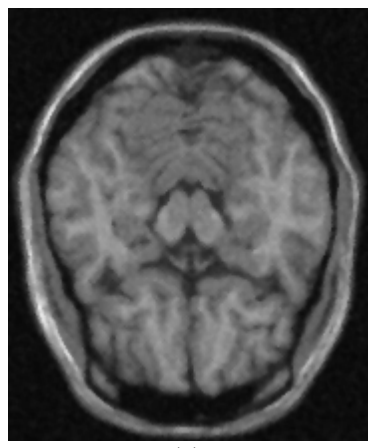

(c)

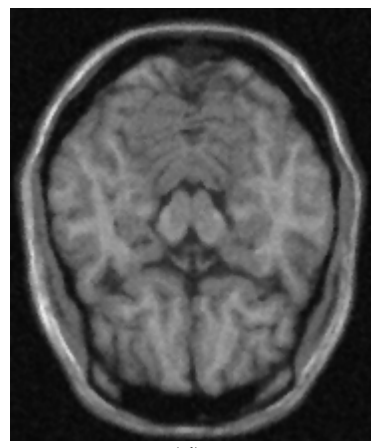

(d)

Fig. 4. Restoration results of the 12 slice in the $5 \mathrm{~mm}$ normal image volume: (a) intact image, (b) 7\% noisy image, (c) restored image of (b) with PSNR $=29.22$ by manually tuned bilateral filtering, (d) restored image of (b) with PSNR = 29.25 using the proposed automatically denoising system.

with the best computation result was obtained while comparing to the manually tuned bilateral filtered result with PSNR $=30.47$. Another restoration example on the $5 \mathrm{~mm}$ normal image was illustrated in Figure 4, where satisfactory results with high PSNR values were obtained. 
Although the PSNR scores by the manually tuned bilateral filter were only slightly lower than the proposed automatically denoising framework, the processing time was dramatically longer. For the proposed approach, the computation time was approximately $3.5 \mathrm{~s}$ for each image on a Windows 7 machine with Intel (R) Core (TM) i5 $2.60 \mathrm{GHz}$ CPU and 8 GB RAM. On the other hand, the manually tuned bilateral filtered results were obtained by 25 times of parameter adjustments. For each trial, the parameter tuning time was roughly $2 \mathrm{~s}$ and the computation time was $0.4 \mathrm{~s}$. In consequence, the ensemble processing time was practically $2.4 \times 25=60 \mathrm{~s}$, not to mention the laborious efforts.

\section{Conclusion}

An artificial neural network based bilateral filter associated with image texture feature analysis has been proposed to automatically denoise brain MR images. In the texture feature analysis, a total of 83 image features were extracted in four different texture feature categories. Based on the paired-samples t-test and the SFS methods, nine optimal features were selected to constitute the minimal subset for training the BPN-based predictable parameter model. A wide variety of MR images with various scenarios were adopted to evaluate the performance of the proposed framework. Experimental results indicated that this new automation method accurately predicted the bilateral filtering parameters and effectively removed the noise in MR images with satisfactory quantity and quality. Comparing to the manually tuned filtering process, our approach not only produced better denoised results but also saved significant processing time. In the future, the proposed framework can be extended to $3 \mathrm{D}$ for volumetric filtering process and potentially with other denoising procedures.

\section{Acknowledgement}

This work was supported by the National Taiwan University under Grant No. NTU-CDP-103R7889.

\section{References}

[1] J.V. Manjón, P. Coupé, L. Concha, A. Buades, D.L. Collins and M. Robles, Diffusion weighted image denoising using overcomplete local PCA, PloS One 8 (2013), e73021.

[2] L. He and R. Greenshields Ian, A nonlocal maximum likelihood estimation method for Rician noise reduction in MR images, IEEE Transactions on Medical Imaging 28 (2009), 165-172.

[3] P. Perona and J. Malik, Scale-space and edge detection using anisotropic diffusion, IEEE Transactions on Pattern Analysis and Machine Intelligence 12 (1990), 629-639.

[4] T. Jerbi, V. Burdin, F. Ghorbel and J.-J. Jacq, Modified data fidelity speed in anisotropic diffusion, 29th Annual International Conference of the IEEE in Engineering in Medicine and Biology Society, Lyon, 2007, pp. 804-807.

[5] C. Tomasi and R. Manduchi, Bilateral filtering for gray and color images, Sixth International Conference on Computer Vision, Bombay, 1998, pp. 839-846.

[6] C.S. Anand and J. Sahambi, MRI denoising using bilateral filter in redundant wavelet domain, TENCON 2008-2008 IEEE Region 10 Conference, Hyderaba, 2008, pp. 1-6.

[7] S.A. Walker, D. Miller and J. Tanabe, Bilateral spatial filtering: Refining methods for localizing brain activation in the presence of parenchymal abnormalities, NeuroImage 33 (2006), 564-569.

[8] I. Frosio, K. Egiazarian and K. Pulli, Machine learning for adaptive bilateral filtering, Image Processing: Algorithms and Systems XIII 9399 (2015), 939908-939908-12.

[9] L. Xiao-zheng, F. Cai-xia and Y. Guang, An MRI image denoising algorithm using neural network analysis and wavelet transformation, Chinese Journal of Magnetic Resonance 25 (2008), 234-242. 
[10] W. Chen, M.L. Giger, H. Li, U. Bick and G.M. Newstead, Volumetric texture analysis of breast lesions on contrast enhanced magnetic resonance images, Magnetic Resonance in Medicine 58 (2007), 562-571.

[11] S. Gao, Y. Peng, H. Guo, W. Liu, T. Gao, Y. Xu, et al., Texture analysis and classification of ultrasound liver images, Bio-Medical Materials and Engineering 24 (2014), 1209-1216.

[12] K. Mala, V. Sadasivam and S. Alagappan, Neural network based texture analysis of CT images for fatty and cirrhosis liver classification, Applied Soft Computing 32 (2015), 80-86.

[13] R.M. Haralick, K. Shanmugam and I.H. Dinstein, Textural features for image classification, IEEE Transactions on Systems, Man and Cybernetics SMC-3 (1973), 610-621.

[14]L.E. George and E.Z. Mohammed, Tissues image retrieval system based on co-occuerrence, run length and roughness features, 2013 International Conference on Computer Medical Applications (ICCMA), Sousse, 2013, pp. 1-6.

[15]C. Sompong and S. Wongthanavasu, MRI brain tumor segmentation using GLCM cellular automata-based texture feature, 2014 International Conference on Computer Science and Engineering (ICSEC), 2014, pp. 192-197.

[16] M.M. Galloway, Texture analysis using gray level run lengths, Computer Graphics and Image Processing 4 (1975), 172179.

[17]X. Tang, Texture information in run-length matrices, IEEE Transactions on Image Processing 7 (1998), 1602-1609.

$[18]$ H. Tamura, S. Mori and T. Yamawaki, Textural features corresponding to visual perception, IEEE Transactions on Systems, Man and Cybernetics 8 (1978), 460-473.

[19]T. Majtner and D. Svoboda, Extension of tamura texture features for 3d fluorescence microscopy, 2012 Second International Conference on 3D Imaging, Modeling, Processing, Visualization and Transmission (3DIMPVT), Zurich, 2012, pp. 301-307.

[20] L. Zong, L. Ying and L. Daxiang, A new texture feature extraction method for image retrieval, 2013 Fourth International Conference on Intelligent Control and Information Processing (ICICIP), 2013, pp. 482-486.

[21] R.A. Fisher, S. Genetiker, S. Genetician, G. Britain and S. Généticien, Statistical Methods for Research Workers, vol. 14 Oliver and Boyd Edinburgh, 1970.

[22] William Sealy Gosset, The probable error of a mean, Biometrika 6 (1908), 1-25.

[23] R.M. Craparo, Significance level, in: Salkind, Neil J. Encyclopedia of Measurement and Statistics, 2007, pp. 889-891.

[24] A.W. Whitney, A direct method of nonparametric measurement selection, IEEE Transactions on Computers 100 (1971), $1100-1103$.

[25]M. Stone, Cross-validatory choice and assessment of statistical predictions, Journal of the Royal Statistical Society, Series B (Methodological) 36 (1974), 111-147.

[26] R. Hecht-Nielsen, Theory of the backpropagation neural network, International Joint Conference on Neural Networks, 1989, pp. 593-605.

[27] D.E. Rumelhart, G.E. Hinton and R.J. Williams, Learning representations by back-propagating errors, Cognitive Modeling 5 (1988), 533-536.

[28] D.E. Rumelhart, J.L. McClelland and P.R. Group, Parallel Distributed Processing 1 (1988), 354-362.

[29] K. Levenberg, A method for the solution of certain problems in least squares, Quarterly of Applied Mathematics 2 (1944), 164-168.

[30] D.W. Marquardt, An algorithm for least-squares estimation of nonlinear parameters, Journal of the Society for Industrial \& Applied Mathematics 11 (1963), 431-441.

[31] BrainWeb: Simulated brain database, available: http://www.bic.mni.mcgill.ca/brainweb/, Nov. 17th, 2014. 